\title{
Cerebral Circulation Time Is a Potential Predictor of Disabling Ischemic Cerebrovascular Events in Patients With Non-disabling Middle Cerebral Artery Stenosis
}

\section{OPEN ACCESS}

Edited by:

QinJian Sun,

Shandong University, China

Reviewed by:

Ahmed Mohamed Elhfnawy, University Hospital Essen, Germany

Nabil Kitchener,

Cairo University, Egypt

*Correspondence:

Xiaobo Wei

xiaobosswei@foxmail.com

Chunguang $\mathrm{Li}$

lichunguang2005@126.com

Qing Wang

denniswq@yahoo.com

†These authors have contributed equally to this work

Specialty section:

This article was submitted to Endovascular and Interventional

Neurology,

a section of the journa

Frontiers in Neurology

Received: 15 January 2021 Accepted: 14 April 2021

Published: 07 May 2021

Citation:

Chen Z, Li M, Wu Z, Zhang M, Weng G, Li M, Liao R, Zhao P, Wu J,

Zhu S, Wang Q, Li C and Wei X (2021)

Cerebral Circulation Time Is a

Potential Predictor of Disabling

Ischemic Cerebrovascular Events in Patients With Non-disabling Middle

Cerebral Artery Stenosis.

Front. Neurol. 12:653752.

doi: 10.3389/fneur.2021.653752

\begin{abstract}
Zhenze Chen ${ }^{1 \dagger}$, Mingchun $\mathrm{Li}^{1+}$, Zhihuan $\mathrm{Wu}^{2+}$, Min Zhang ${ }^{3}$, Guomei Weng ${ }^{2}$, Minzi $\mathrm{Li}^{1}$,
\end{abstract} Rongxin Liao ${ }^{4}$, Peng Zhao ${ }^{4}$, Jianming $W_{u^{4}}$, Shuzhen $Z \mathrm{Zhu}^{1}$, Qing Wang ${ }^{1 *}$, Chunguang $\mathrm{Li}^{1 *}$ and Xiaobo Wei ${ }^{1 *}$

${ }^{1}$ Department of Neurology, Zhujiang Hospital, Southern Medical University, Guangzhou, China, ${ }^{2}$ Department of Neurology, 1st People Hospital of Zhaoqing, Zhaoqing, China, ${ }^{3}$ Department of Neurology, Jiangmen Centrol Hospital, Jiangmen, China, ${ }^{4}$ Department of Gerontology, Integrated Hospital of Traditional Chinese Medicine, Southern Medical University, Guangzhou, China

Patients with non-disabling middle cerebral artery (MCA) stenosis (ND-MCAS) are at risk for disabling ischemic cerebrovascular events (DICE) despite aggressive medical therapy. In this study, we aimed to verify whether cerebral circulation time (CCT) was a potential predictor of DICE in patients with ND-MCAS. From January 2015 to January 2020, 46 patients with ND-MCAS treated with aggressive medical therapy were enrolled for digital subtraction angiography (DSA) in this convenience sampling study. They were divided into the DICE (-) and DICE (+) groups based on the occurrence of DICE within 3 months after DSA. The CCT was defined as the time from the appearance of the MCA to the peak intensity of the Trolard vein during DSA. The rCCT (relative CCT) was defined as the ratio of the CCT of the stenotic side (SCCT) to the CCT of the healthy side (hCCT). The differences in SCCT, hCCT, and rCCT between the two groups were analyzed with Mann-Whitney $U$ tests. Logistic regression analysis was performed to evaluate the association between the risk factors and DICE. Receiver operating characteristic (ROC) curves were constructed to assess the predictive value of rCCT in identifying DICE in ND-MCAS patients. The results showed that DICE appeared in 5 of the 46 patients within 3 months. rCCT were significantly increased in the DICE $(+)$ group compared with the DICE (-) group $[1.08(1.05,1.14)$ vs. $1.30(1.22,1.54), p<0.001]$. Logistic regression analysis found that prolonged $\mathrm{rCCT}$ was an independent positive prognostic factor for DICE (odds ratio $=1.273, p=0.019$ ) after adjustment for potential confounders (age, diabetes, antithrombotic use, and stenosis degree). ROC analysis showed that $\mathrm{rCCT}$ provided satisfactory accuracy in distinguishing the DICE (+) group from the DICE (-) group among ND-MCAS patients (area under the curve $=0.985, p<0.001$ ), with an optimal cutoff point of 1.20 (100\% sensitivity, $97.6 \%$ specificity). In conclusion, prolonged rCCT is independently associated with the occurrence of DICE in ND-MCAS patients and may be used to identify individuals at risk of DICE.

Keywords: non-disabling middle cerebral artery stenosis, cerebral circulation time, digital subtraction angiography, disabling ischemic cerebrovascular events, prognosis 


\section{INTRODUCTION}

Stenting, angioplasty and aggressive medical management are established procedures for the prevention of further ischemic events following middle cerebral artery (MCA) stenosis (1-6). In recent years, an increasing proportion of patients with nondisabling MCA stenosis (ND-MCAS) have been treated with dual antiplatelet agents (7). The evidence to support their use comes mainly from the results of the two randomized clinical trials Stenting and Aggressive Medical Management for Preventing Recurrent Stroke in Intracranial Stenosis (SAMMPRIS) and Vitesse Intracranial Stent Study for Ischemic Stroke Therapy (VISSIT), which showed that pharmacologic management is safer and has a lower incidence of ischemic events than stenting $(8,9)$. However, SAMMPRIS and VISSIT showed a 5.8 and $9.4 \%$ risk of stroke and death, respectively, at 30 days during the treatment with dual antiplatelet agents $(2,8,9)$. Therefore, early identification and subsequent stenting or angioplasty should be carried out for patients who are at high risk of disabling ischemic cerebrovascular events (DICE) or death.

Impaired cerebrovascular reserve is an important predictor of stroke and transient ischemic attack (TIA) in patients with cerebral artery stenosis or occlusion (10-12). At present, the clinical evaluation of the cerebrovascular reserve mainly relies on acetazolamide-challenged single-photon emission computed tomography (ACZ-challenged SPECT), computed tomography perfusion imaging (CTP) and magnetic resonance (MR) perfusion-weighted imaging (PWI), which are expensive and expose the patients to radiation and contrast medium (1315). The cerebral circulation time (CCT) derived from digital subtraction angiography (DSA) has been reported to be wellcorrelated with cerebrovascular reserve $(12,16-21)$. It can help surgeons observe the patient's cerebrovascular reserve during surgery without the need for SPECT, CTP, and PWI.

In this study, we carried out a retrospective analysis of all prospectively collected data from patients with ND-MCAS treated with aggressive medical therapy, and aimed to verify whether CCT could be used as a potential predictor of DICE for patients with ND-MCAS.

\section{MATERIALS AND METHODS}

\section{Patients and Study Design}

The clinical and radiological data from 273 patients with MCA stenosis treated at Zhujiang Hospital of Southern Medical University, Guangzhou, China between January 2015 and January 2020 were reviewed in this convenience sampling study. After excluding 227 patients with disability or multiple vascular stenosis, we enrolled 46 patients with non-disabling unilateral MCA stenosis in the final analysis. Their demographic characteristics, stroke risk factors, clinical symptoms, medications and relevant scale scores [National Institutes of National Institutes of Health Stroke Scale (NIHSS) and Modified Rankin Scale (mRS)] were reviewed by two neurologists. According to the trial A Pooled Analysis of Clopidogrel in High-Risk Patients with Acute Non-Disabling Cerebrovascular Events (CHANCE), non-disabling ischemic stroke was defined as: 1. Transient ischemic attack (TIA); 2. Minor ischemic stroke $(22,23)$. Transient ischemic attack was defined as a transient episode of neurological symptoms caused by focal cerebral or retinal ischemia without radiographic evidence of acute infarction (24). Minor ischemic stroke was defined as a stroke with a NIHSS score $\leq 3$ or mRS score $\leq 3$ (25). Patients with ND-MCAS were allocated to the DICE (+) group if they suffered from disabling ischemic cerebrovascular events (NIHSS score $>3$ or mRS score $>3$ ) in 90 days after the onset of the stroke. Otherwise, patients were allocated to the DICE (-) group. This study was approved by the Ethics Committee of Zhujiang Hospital of Southern Medical University and conducted in accordance with the ethical standards of the 1975 Declaration of Helsinki and the 1999 National Institutes of Health Human Subjects Policies and Guidance.

\section{DSA Protocol and Data Analysis}

All patients were enrolled for DSA with a uniform and standard protocol in Zhujiang Hospital of Southern Medical University, Guangzhou, Guangdong, China. During the DSA procedures, a $5 \mathrm{~F}$ angio-catheter was placed in the carotid artery at the $\mathrm{C} 3$ vertebral body level. All of the subjects were examined by a single-plane angiographic machine (GE IGS 330, America) with a power injector $(5 \mathrm{ml}$ ioversol injection with $3 \mathrm{ml} / \mathrm{s}$ speed, 150 $\mathrm{psi} / \mathrm{kg}$ pressure). The stenotic degree was measured according to the NASCET criteria from DSA image (26). Neuroimaging and analysis were performed independently by two trained neurologists who were blinded to the clinical conditions and were responsible for assessing all of the neuroimaging variables used in this study.

\section{Cerebral Circulation Time Measurement}

During the angiography procedure, the CCT was defined as the time from the appearance of the MCA to the peak intensity of the Trolard vein (Figure 1). In order to reduce the data errors, the CCT of same patient was measured for three times, and then the average of these three measurements was used in the following analysis. The main research indicator of the present study was rCCT (relative CCT), which was calculated with the following formula: $\mathrm{rCCT}=$ the CCT of the stenotic side $(\mathrm{sCCT}) /$ the CCT of the healthy side (hCCT).

\section{Treatment Options}

All patients received aggressive medical therapy for at least 3 months after the DSA examination. Four of the 5 patients in the DICE (+) group and 27 of the 41 patients in the DICE (-) group received dual antiplatelet therapy (100 $\mathrm{mg}$ aspirin and $75 \mathrm{mg}$ clopidogrel per day). The remaining patients all received single antiplatelet therapy. Patients suffering from hypertension, diabetes, or dyslipidemia all received standard drug treatment.

\section{Statistical Analysis}

All statistical analyses were conducted using SPSS 23 for Windows. All continuous variables were tested for normality and expressed as median (interquartile range). Categorical variables were expressed as numbers (\%). The differences in demographic and clinical variables between the DICE $(-)$ and DICE $(+)$ 


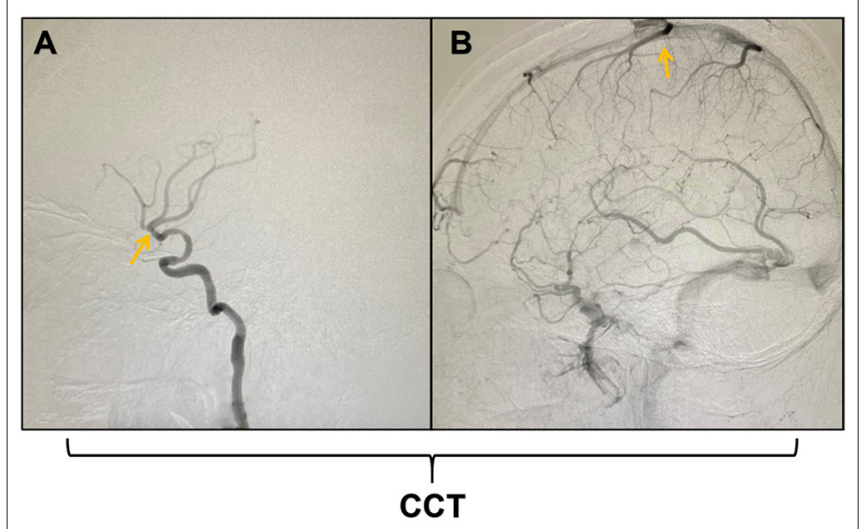

FIGURE 1 | Schematic diagram of the measurement of cerebral circulation time (CCT). (A) shows the appearance of the middle cerebral artery (MCA); (B) shows the peak intensity of the Trolard vein. The CCT was defined as the time from the appearance of the MCA to the peak intensity of the Trolard vein during digital subtraction angiography (DSA).

groups were analyzed by Mann-Whitney U-tests for continuous variables and Pearson's $\chi^{2}$ test or Fisher's exact test for categorical variables. Logistic regression analysis was performed to estimate the odds ratio (OR) for DICE with rCCT and other risk factors as independent variables. $P<0.05$ was considered statistically significant. Receiver operating characteristic (ROC) curve analysis of rCCT was performed to evaluate the predictive accuracy of DICE in the ND-MCAS patients.

\section{RESULTS}

Between January 2015 and January 2020, 46 patients with NDMCAS were confirmed by DSA in our hospital, including 30 males $(65.2 \%)$, with an average age of $55(48.7,63)$ (range $25-$ 87 years), hypertension (45.6\%), diabetes (26.1\%), dyslipidemia (45.6\%) and smoking (26.1\%). Eighteen of the patients presented with TIA, and 28 presented with minor ischemic stroke. Among all of the included patients, 5 patients suffered from DICE within 3 months after DSA and were allocated to the DICE (+) group, and the remaining patients without disability were allocated to the DICE (-) group. The demographic and clinical data of the patients is shown in Table 1.

No significant differences were observed in sex, stroke risk factors, baseline clinical manifestation or usage of drugs between the DICE (-) and DICE $(+)$ groups. The rCCT in the DICE $(+)$ group were prolonged compared with those in the DICE (-) group $[1.08(1.05,1.14)$ vs. $1.30(1.22,1.54), p<0.001$; Table 1 , Figure 2]. There was no significant difference in sCCT and hCCT between the two groups $[6.83(6.50,7.75)$ vs. $7.67(6.50,10.67)$, $p=0.258 ; 6.33(5.92,7.00)$ vs. 6.25 (4.96, 7.25), $p=0.480$;

\section{Table 1, Figure 2].}

Logistic regression analysis showed that after adjustment for possible confounders, namely, age, diabetes, stenosis degree and antithrombotic use, a longer rCCT was independently associated with a higher risk of DICE $(\mathrm{OR}=1.273, p=0.019$, Table 2$)$. The
TABLE 1 | The demographic and clinical data for patients with and without DICE.

\begin{tabular}{|c|c|c|c|}
\hline Characteristics & $\operatorname{DICE}(-)(N=41)$ & DICE $(+)(N=5)$ & $p$-value \\
\hline Age (years) & $54(47.5,61)$ & $69(57.5,77)^{\star}$ & 0.026 \\
\hline Male & $28(68.3)$ & $2(40)$ & 0.449 \\
\hline Hypertension & $19(46.3)$ & $2(40)$ & 1 \\
\hline Diabetes & $9(22.5)$ & $3(60)$ & 0.197 \\
\hline Dyslipidemia & $18(43.9)$ & $3(60)$ & 0.836 \\
\hline Cigarette smoking & $11(26.8)$ & $1(20)$ & 1 \\
\hline \multicolumn{4}{|c|}{ Usage of drugs within 3 months } \\
\hline Dual antiplatelet agents & $27(65.9)$ & $4(80)$ & 0.895 \\
\hline Intensive lipid-lowering & $13(31.7)$ & $2(40)$ & 1 \\
\hline \multicolumn{4}{|c|}{ Clinical manifestation and score } \\
\hline Transient ischemic attack & $15(36.5)$ & $3(60)$ & 0.598 \\
\hline minor ischemic stroke & $26(63.5)$ & $2(40)$ & \\
\hline NIHSS (baseline) & $0(0,1)$ & $0(0,1)$ & 0.587 \\
\hline NIHSS (3 month) & $0(0,1)$ & $6(4.5,8.5)^{\star \star \star}$ & $<0.001$ \\
\hline mRs (baseline) & $1(0,1)$ & $0(0,1)$ & 0.233 \\
\hline mRs (3 month) & $1(0,1)$ & $3(2.5,4)^{\star \star \star}$ & $<0.001$ \\
\hline \multicolumn{4}{|l|}{ Image data } \\
\hline Stenosis degree (\%) & $70(30,80)$ & $80(60,85)$ & 0.391 \\
\hline sCCT (s) & $6.83(6.50,7.75)$ & $7.67(6.50,10.67)$ & 0.258 \\
\hline hCCT (s) & $6.33(5.92,7.00)$ & $6.25(4.96,7.25)$ & 0.480 \\
\hline rCCT & $1.08(1.05,1.14)$ & $1.30(1.22,1.54)^{\star \star \star}$ & $<0.001$ \\
\hline
\end{tabular}

${ }^{*} p<0.05$ vs. DICE (-) group. ${ }^{* \star *} p<0.001$ vs. DICE (-) group.

All continuous variables are expressed as median (interquartile range) and categorical variables are expressed as numbers (\%). The statistically significant differences between the DICE (-) and DICE (+) groups were assessed by $\chi^{2}$-test or Mann-Whitney Utests. DICE, disabling ischemic cerebrovascular events; NIHSS, National Institutes of National Institutes of Health Stroke Scale; mRs, The Modified Rankin Scale; CCT, cerebral circulation time; sCCT, CCT of stenotic side; hCCT, CCT of healthy side; rCCT, relative $C C T=$ sCCT/hCCT.

ROC curves of CCT and the other risk factors for the prediction of DICE are shown in Figure 3. The optimal cutoff point of rCCT (1.20) predicted DICE with $100 \%$ sensitivity and $97.6 \%$ specificity (Table 3, Figure 3). In contrast, the optimal cutoff point of sCCT (9.5 s) predicted DICE with $40 \%$ sensitivity and $100 \%$ specificity; the optimal cutoff point of stenosis degree (65\%) predicted DICE with $100 \%$ sensitivity and $43.9 \%$ specificity (Table 3, Figure 3).

\section{DISCUSSION}

Non-disabling ischemic stroke, which indicates a patient is at risk of an early recurrent stroke, can lead to severe disabling events and even death, seriously affecting their quality of life (27-30). Therefore, early identification of such patients is particularly important (31-33). In this study, we provided novel observations by evaluating the usefulness of CCT for the prediction of DICE in ND-MCAS patients.

Compared with other previous studies, we specifically demonstrated the presence of prolonged rCCT in arterial stenosis. Yamamoto et al. detected prolonged CCT measured through visual observation in patients with unilateral occlusive lesions in the ICA or MCA (21). Lin et al. investigated multiple segments of CCT in 25 patients with carotid stenosis and 34 
A

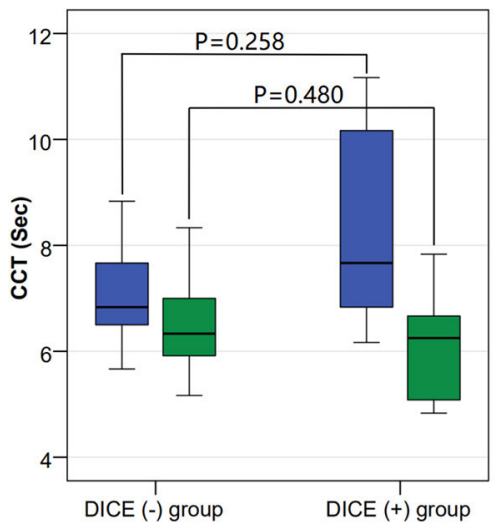

B

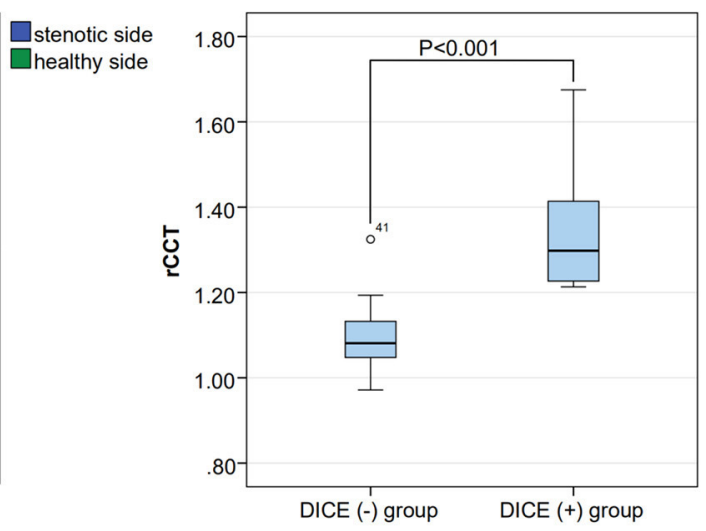

FIGURE 2 | Comparison of the stenotic side CCT (SCCT), the healthy side CCT (hCCT) and the relative CCT (rCCT) among the DICE (-) and DICE (+) groups. (A) shows no difference in hCCT [6.33 (5.92, 7.00) vs. $6.25(4.96,7.25), p=0.480]$ and sCCT $[6.83(6.50,7.75)$ vs. $7.67(6.50,10.67), p=0.258]$ between the DICE $(-)$ and DICE $(+)$ groups. (B) shows a significant difference in rCCT $(1.37 \pm 0.19$ vs. $1.09 \pm 0.07, p<0.001)$ between the DICE $(-)$ and DICE $(+)$ groups.

TABLE 2 | Multivariable logistic regression analysis to evaluate the association between DICE and risk factors, including rCCT.

\begin{tabular}{|c|c|c|c|c|c|c|}
\hline \multirow[t]{2}{*}{ Variables } & \multicolumn{3}{|c|}{ Univariate } & \multicolumn{3}{|c|}{ Multivariate model } \\
\hline & OR & $95 \% \mathrm{Cl}$ & $p$-value & OR & $95 \% \mathrm{Cl}$ & $p$-value \\
\hline rCCT & 1.295 & $1.053-1.592$ & 0.014 & $1.273^{*}$ & $1.041-1.556$ & 0.019 \\
\hline $\begin{array}{l}\text { Age (years) } \\
\text { (mean } \pm S D)\end{array}$ & 1.093 & $1.004-1.190$ & 0.040 & 1.063 & $0.865-1.306$ & 0.564 \\
\hline $\begin{array}{l}\text { Dual antiplatelet } \\
\text { therapy }\end{array}$ & 0.482 & $0.049-4.735$ & 0.531 & 1.646 & $0.006-455.233$ & 0.862 \\
\hline Stenosis degree & 1.025 & $0.978-1.075$ & 0.297 & 1.052 & $0.857-1.292$ & 0.626 \\
\hline Diabetes & 5.333 & 0.769-36.965 & 0.090 & 0.127 & 0.002-8.309 & 0.333 \\
\hline
\end{tabular}

${ }^{*} p<0.05$.

DICE, disabling ischemic cerebrovascular events; CCT, cerebral circulation time; rCCT, relative $C C T=S C C T / h C C T ; O R$, odds ratio; $C l$, confidence interval.

normal control subjects and found that some of the segments of CCT were longer in patients with carotid stenosis than in control subjects (34). In our study, we found that $\mathrm{rCCT}$ was a more sensitive marker than sCCT for predicting DICE in ND-MCAS.

Impaired cerebrovascular reserve is the main cause of cerebral infarction. Lin et al. showed that CBF, MTT and Tmax were found to correlate with CCT. Prolonged CCT may reflect the impairment of cerebrovascular reserve (20). This conclusion is in line with our findings, in which 5 of the 46 patients showed significant prolongation of rCCT and subsequently suffered DICE. The rCCT in these 5 patients were significantly increased compared with the patients without DICE. We could conclude that rCCT may reflect the cerebrovascular reserve and predict the occurrence of DICE. At the same time, possibly because rCCT excluded the difference in CCT among individuals, rCCT was found to be more effective than sCCT in predicting DICE (AUC of $\mathrm{rCCT}=0.985, p<0.001$, AUC of $\mathrm{sCCT}=0.656, p<0.259)$.

Our results also suggested that $\operatorname{rCCT}(\mathrm{OR}=1.273, p=$ 0.019) was a more sensitive predictor of DICE than the degree

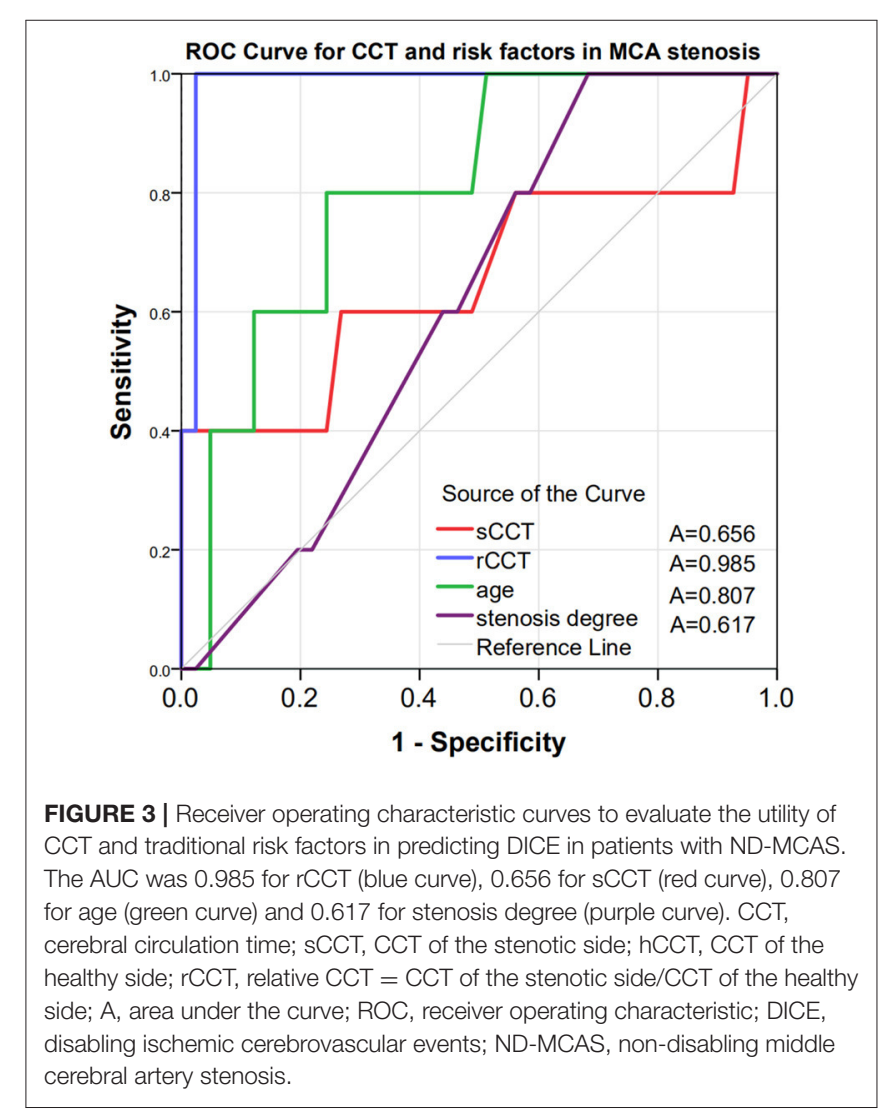

of stenosis $(\mathrm{OR}=1.025, p=0.626)$ after adjustment for age, diabetes, usage of dual antiplatelet therapy and stenosis degree. Yong's study showed that prolonged CCT was more closely associated with symptomatic carotid stenosis than stenosis degree or collateral circulation (34). This theory also seems to be applicable in our study for intracranial vascular stenosis. 
TABLE 3 | ROC curves for traditional risk factors and CCT in the prediction of disabling ischemic cerebrovascular events.

\begin{tabular}{|c|c|c|c|c|}
\hline \multirow[t]{2}{*}{ Variable } & \multicolumn{2}{|c|}{ Traditional risk factors } & \multicolumn{2}{|c|}{ СCT } \\
\hline & Age & Stenosis degree & sCCT & rCCT \\
\hline$A \cup C$ & $0.807^{\star}$ & 0.617 & 0.656 & $0.985^{\star \star \star}$ \\
\hline Cutoff value & 0.556 & 0.439 & 0.4 & 0.976 \\
\hline$p$-value & 0.026 & 0.397 & 0.259 & $<0.001$ \\
\hline $95 \% \mathrm{Cl}$ & $0.636-0.979$ & $0.417-0.817$ & $0.338-0.974$ & $0.953-1.000$ \\
\hline Sensitivity & $80 \%$ & $100 \%$ & $40 \%$ & $100 \%$ \\
\hline Specificity & 75.6 & $43.9 \%$ & $100 \%$ & $97.6 \%$ \\
\hline
\end{tabular}

${ }^{*} p<0.05,{ }^{* * *} p<0.001$.

$R O C$, receiver operating characteristic; $C C T$, cerebral circulation time; rCCT, relative CCT = sCCT/hCCT; AUC, area under the curve; Cl, confidence interval.

Scientific statements from the American Heart Association have shown that angioplasty or placement of a Wingspan stent may be warranted for patients with severe stenosis (70$99 \%$ ) of a major intracranial artery who have progressing symptoms, recurrent TIA or stroke (2). In this study, the risk of DICE occurring in patients with MCA stenosis was assessed in real-time by intraoperative measurement of rCCT. For patients with severe rCCT prolongation, traditional drug therapy may not prevent the occurrence of severe DICE. Thus, with the help of rCCT in the prediction of DICE, the surgeons may prefer immediate intravascular treatment after calculating rCCT, avoiding the additional radiation, contrast agent and economic burden from SPECT, CTP, PWI, and secondary surgery.

The limitations of this study include the small number of cases and the imbalance in the number of cases between the DICE $(-)$ and DICE (+) groups. The patients in the present study were followed up for only 3 months. Longitudinal studies of large cohorts and longer follow-up periods are needed to confirm our results. In addition, collateral circulation may influence the prognosis of the patients (35). The relationship between CCT and collateral circulation was not included in the analysis of this study, and it may need further investigation in the future.

\section{CONCLUSIONS}

Prolonged rCCT is an independent positive prognostic factor for the occurrence of DICE in patients with ND-MCAS treated with drugs, and it could be used by surgeons to identify individuals at high risk of DICE during surgery. In patients with a significantly prolonged CCT, angioplasty or placement of a balloon expandable and self-expanding vascular stents may be warranted even when their degree of stenosis is $<70 \%$. Future studies with larger sample sizes are required to further verify the sensitivity and accuracy of CCT in the clinical management of stroke.

\section{DATA AVAILABILITY STATEMENT}

The raw data supporting the conclusions of this article will be made available by the authors, without undue reservation.

\section{ETHICS STATEMENT}

The studies involving human participants were reviewed and approved by the Ethics Committee of Zhujiang Hospital of Southern Medical University and conducted in accordance with the ethical standards of the 1975 Declaration of Helsinki and the 1999 National Institutes of Health Human Subjects Policies and Guidance. The patients/participants provided their written informed consent to participate in this study.

\section{AUTHOR CONTRIBUTIONS}

ZC, XW, CL, and QW: conceived and designed the clinical study. ZC, MingL, ZW, MZ, MinzL, GW, SZ, CL, XW, and QW: performed the clinical study. ZC, CL, XW, and QW: analyzed the data. CL, XW, and QW: revised the paper for intellectual content. ZC, CL, XW, and QW: wrote the paper. All authors read and approved the final manuscript.

\section{FUNDING}

This work was supported by the Natural Science Foundations of Guangdong of China (Grant No: 2019A1515010281), Guangdong Provincial Key Laboratory of Shock and Microcirculation, Initiated Foundation of Zhujiang Hospital (Grant No: 02020318005), Science and Technology Program of Guangdong of China (No: 2020A0505100037), and Leading Talent in Talents Project Guangdong High-level Personnel of Special Support Program to QW; and National Natural Science Foundation of China (Grant No: 81801252) to XW.

\section{ACKNOWLEDGMENTS}

We thank all of the participants for their valuable contribution to this research and managing the data for this project.

\section{REFERENCES}

1. Chimowitz M, Lynn M, Howlett-Smith H, Stern B, Hertzberg V, Frankel M, et al. Comparison of warfarin and aspirin for symptomatic intracranial arterial stenosis. N Engl J Med. (2005) 352:1305-16. doi: 10.1056/NEJMoa043033

2. Eskey C, Meyers P, Nguyen T, Ansari S, Jayaraman M, McDougall C, et al. Indications for the performance of intracranial endovascular neurointerventional procedures: a scientific statement from the American Heart Association. Circulation. (2018) 137:e661-89. doi: 10.1161/CIR.0000000000000567 
3. Kernan W, Ovbiagele B, Black H, Bravata D, Chimowitz M, Ezekowitz M, et al. Guidelines for the prevention of stroke in patients with stroke and transient ischemic attack: a guideline for healthcare professionals from the American Heart Association/American Stroke Association. Stroke. (2014) 45:2160-236. doi: 10.1161/STR.0000000000000024

4. Liu S, Zheng H, Yu W, Ramakrishnan V, Shah S, Gonzalez L, et al. Investigation of S-Nitrosoglutathione in stroke: a systematic review and metaanalysis of literature in pre-clinical and clinical research. Exp Neurol. (2020) 328:113-262. doi: 10.1016/j.expneurol.2020.113262

5. Ni L, Zhou F, Qing Z, Zhang X, Li M, Zhu B, et al. The asymmetry of white matter hyperintensity burden between hemispheres is associated with intracranial atherosclerotic plaque enhancement grade. Front Aging Neurosci. (2020) 12:163. doi: 10.3389/fnagi.2020.00163

6. Shetty A, Upadhya R, Madhu L, Kodali M. Novel insights on systemic and brain aging, stroke, amyotrophic lateral sclerosis, and Alzheimer's disease. Aging Dis. (2019) 10: 470-82. doi: 10.14336/AD.2019.0330

7. Turan T, Cotsonis G, Lynn M, Wooley R, Swanson S, Williams J, et al. Intracranial stenosis: impact of randomized trials on treatment preferences of US neurologists and neurointerventionists. Cerebrovasc Dis. (2014) 37:20311. doi: $10.1159 / 000358120$

8. Chimowitz M, Lynn M, Derdeyn C, Turan T, Fiorella D, Lane B, et al. Stenting versus aggressive medical therapy for intracranial arterial stenosis. $\mathrm{N} \mathrm{Engl} \mathrm{J}$ Med. (2011) 365:993-1003. doi: 10.1056/NEJMoa1105335

9. Zaidat O, Fitzsimmons B, Woodward B, Wang Z, Killer-Oberpfalzer M, Wakhloo A, et al. Effect of a balloon-expandable intracranial stent vs medical therapy on risk of stroke in patients with symptomatic intracranial stenosis: the VISSIT randomized clinical trial. JAMA. (2015) 313:1240-8. doi: 10.1001/jama.2015.1693

10. Markus H, Cullinane M. Severely impaired cerebrovascular reactivity predicts stroke and TIA risk in patients with carotid artery stenosis and occlusion. Brain. (2001) 124:457-67. doi: 10.1093/brain/124.3.457

11. McKetton L, Cohn M, Tang-Wai D, Sobczyk O, Duffin J, Holmes K, et al. Cerebrovascular resistance in healthy aging and mild cognitive impairment. Front Aging Neurosci. (2019) 11:79. doi: 10.3389/fnagi.20 19.00079

12. Riou-Comte N, Guillemin F, Gory B, Lapergue B, Zhu F, Soudant M, et al. Predictive factors of functional independence after optimal reperfusion in anterior circulation ischaemic stroke with indication for intravenous thrombolysis plus mechanical thrombectomy. Eur J Neurol. (2021) 28:141-51. doi: 10.1111/ene.14509

13. Doerfler A, Eckstein H, Eichbaum M, Heiland S, Benner T, Allenberg J, et al. Perfusion-weighted magnetic resonance imaging in patients with carotid artery disease before and after carotid endarterectomy. J Vasc Surg. (2001) 34:587-93. doi: 10.1067/mva.2001.118588

14. Leinsinger G, Piepgras A, Einhäupl K, Schmiedek P, Kirsch C. Normal values of cerebrovascular reserve capacity after stimulation with acetazolamide measured by xenon 133 single-photon emission CT. AJNR Am J Neuroradiol. (1994) 15:1327-32.

15. Wintermark M, Fischbein N, Smith W, Ko N, Quist M, Dillon W. Accuracy of dynamic perfusion CT with deconvolution in detecting acute hemispheric stroke. AJNR Am J Neuroradiol. (2005) 26:104-12.

16. Bashir A, Abebe Z, McInnes K, Button E, Tatarnikov I, Cheng W, et al. Increased severity of the CHIMERA model induces acute vascular injury, sub-acute deficits in memory recall, and chronic white matter gliosis. Exp Neurol. (2020) 324:113-6. doi: 10.1016/j.expneurol.2019. 113116

17. Cramer N, Korotcov A, Bosomtwi A, Xu X, Holman D, Whiting K, et al. Neuronal and vascular deficits following chronic adaptation to high altitude. Exp Neurol. (2019) 311:293-304. doi: 10.1016/j.expneurol.2018. 10.007

18. Guenego A, Fahed R, Albers G, Kuraitis G, Sussman E, Martin B, et al. Hypoperfusion intensity ratio correlates with angiographic collaterals in acute ischaemic stroke with M1 occlusion. Eur J Neurol. (2020) 27:864-70. doi: 10.1111/ene.14181

19. Aikawa H, Kazekawa K, Tsutsumi M, Onizuka M, Iko M, Kodama T, et al. Intraprocedural changes in angiographic cerebral circulation time predict cerebral blood flow after carotid artery stenting. Neurol Med Chir. (2010) 50:269-74. doi: 10.2176/nmc.50.269
20. Lin C, Hung S, Chang F, Guo W, Luo C, Kowarschik M, et al. Finding the optimal deconvolution algorithm for MR perfusion in carotid stenosis: correlations with angiographic cerebral circulation time. J Neuroradiol. (2016) 43:290-6. doi: 10.1016/j.neurad.2016.02.002

21. Yamamoto S, Watanabe M, Uematsu T, Takasawa K, Nukata M, Kinoshita N. Correlation of angiographic circulation time cerebrovascular reserve by acetazolamide-challenged single photon emission CT. AJNR Am J Neuroradiol. (2004) 25:242-7.

22. Pan Y, Elm J, Li H, Easton J, Wang Y, Farrant M, et al. Outcomes associated with clopidogrel-aspirin use in minor stroke or transient ischemic attack: a pooled analysis of clopidogrel in high-risk patients with acute nondisabling cerebrovascular events (CHANCE) and platelet-oriented inhibition in new TIA and minor ischemic stroke (POINT) trials. JAMA Neurol. (2019) 76:1466-73. doi: 10.1001/jamaneurol.2019.2531

23. Wang Y, Wang Y, Zhao X, Liu L, Wang D, Wang C, et al. Clopidogrel with aspirin in acute minor stroke or transient ischemic attack. N Engl J Med. (2013) 369:11-9. doi: 10.1056/NEJMoa1215340

24. Easton J, Saver J, Albers G, Alberts M, Chaturvedi S, Feldmann E, et al. Definition and evaluation of transient ischemic attack: a scientific statement for healthcare professionals from the American Heart Association/American Stroke Association Stroke Council; Council on Cardiovascular Surgery and Anesthesia; Council on Cardiovascular Radiology and Intervention; Council on Cardiovascular Nursing; and the Interdisciplinary Council on Peripheral Vascular Disease. The American Academy of Neurology affirms the value of this statement as an educational tool for neurologists. Stroke. (2009) 40:2276-93. doi: 10.1161/STROKEAHA.108.192218

25. Johnston S, Elm J, Easton J, Farrant M, Barsan W, Kim A, et al. Time course for benefit and risk of clopidogrel and aspirin after acute transient ischemic attack and minor ischemic stroke. Circulation. (2019) 140:658-64. doi: 10.1161/CIRCULATIONAHA.119.040713

26. Saba L, Mallarini G. A comparison between NASCET and ECST methods in the study of carotids: evaluation using multi-detector-row CT angiography. Eur J Radiot. (2010) 76:42-7. doi: 10.1016/j.ejrad.2009.04.064

27. Cheng Z, Geng X, Gao J, Hussain M, Moon S, Du H, et al. Intravenous administration of standard dose tirofiban after mechanical arterial recanalization is safe and relatively effective in acute ischemic stroke. Aging Dis. (2019) 10:1049-57. doi: 10.14336/AD.2018.0922

28. Wang Z, Wang C, Zhang P, Qu Y, Guo Z, Yang Y, et al. Effects of early changes in blood pressure during intravenous thrombolysis on the prognosis of acute ischemic stroke patients. Front Aging Neurosci. (2020) 12:601471. doi: 10.3389/fnagi.2020.601471

29. Wu L, Huber M, Wu D, Chen J, Li M, Ding Y, et al. Intra-arterial cold saline infusion in stroke: historical evolution and future prospects. Aging Dis. (2020) 11:1527-36. doi: 10.14336/AD.2020.0325

30. Zhu S, Wei X, Yang X, Huang Z, Chang Z, Xie F, et al. Plasma lipoprotein-associated phospholipase $\mathrm{A} 2$ and superoxide dismutase are independent predicators of cognitive impairment in cerebral small vessel disease patients: diagnosis and assessment. Aging Dis. (2019) 10:834-46. doi: 10.14336/AD.2019.0304

31. Li W, Qi Z, Kang H, Qin X, Song H, Sui X, et al. Serum occludin as a biomarker to predict the severity of acute ischemic stroke, hemorrhagic transformation, and patient prognosis. Aging Dis. (2020) 11:1395-406. doi: 10.14336/AD.2020.0119

32. Viderman D, Issanov A, Temirov $\mathrm{T}$, Goligher $\mathrm{E}$, la Fleur $\mathrm{P}$. Outcome predictors of stroke mortality in the neurocritical care unit. Front Neurol. (2020) 11:579733. doi: 10.3389/fneur.2020. 579733

33. Zhang S, Cho J, Nguyen T, Spincemaille P, Gupta A, Zhu W, et al. Initial experience of challenge-free MRI-based oxygen extraction fraction mapping of ischemic stroke at various stages: comparison with perfusion and diffusion mapping. Front Neurosci. (2020) 14:535441. doi: 10.3389/fnins.2020.5 35441

34. Lin CJ, Hung SC, Guo WY, Chang FC, Luo CB, Beilner J, et al. Monitoring peri-therapeutic cerebral circulation time: a feasibility study using color-coded quantitative DSA in patients with steno-occlusive arterial disease. AJNR Am J Neuroradiol. (2012) 33:1685-90. doi: 10.3174/ajnr.A3049

35. Hu YS, Guo WY, Lee IH, Chang FC, Lin CJ, Luo CB, et al. Prolonged cerebral circulation time is more associated with symptomatic 
carotid stenosis than stenosis degree or collateral circulation. $J$ Neurointervent Surg. (2018) 10:476-80. doi: 10.1136/neurintsurg-2017-0 13293

Conflict of Interest: The authors declare that the research was conducted in the absence of any commercial or financial relationships that could be construed as a potential conflict of interest.
Copyright $\odot 2021$ Chen, Li, Wu, Zhang, Weng, Li, Liao, Zhao, Wu, Zhu, Wang, Li and Wei. This is an open-access article distributed under the terms of the Creative Commons Attribution License (CC BY). The use, distribution or reproduction in other forums is permitted, provided the original author(s) and the copyright owner(s) are credited and that the original publication in this journal is cited, in accordance with accepted academic practice. No use, distribution or reproduction is permitted which does not comply with these terms. 